\title{
Brucellosis induced avascular necrosis of the femoral head in a 7 year old child
}

\author{
Abraham Gedalia, Charles Howard, Menachem Einhorn
}

\begin{abstract}
The case is reported of a 7 year old Bedouin girl who presented with a three week history of fever and limp. Initial tests for rheumatoid factor, antinuclear antibodies, and brucella were negative. Two weeks after admission Brucella melitensis was cultured from bone marrow and synovial fluid samples. The patient improved after treatment with doxycillin and streptomycin, but radiographs of the right hip showed avascular necrosis of the right femoral head. A mild limp and limitation of motion in the right hip persisted 18 months after admission.
\end{abstract}

Brucellosis is endemic among the Bedouins in the Negev region of Israel. ${ }^{1}$ Brucella melitensis is responsible for the disease in this area. ${ }^{2}$ The clinical spectrum of brucellosis ranges from very mild, febrile illness to severe multisystem disease. It may follow an acute, subacute, or chronic course. Osteoarticular manifestations are common in brucellosis, usually presenting as arthritis. Osteomyelitis due to brucella infection is unusual in adults and rare in children. ${ }^{3}$ This report describes a child with acute brucellosis presenting with monarticular arthritis of the hip, whose disease course was complicated by avascular necrosis causing destruction of the femoral head.

\section{Case report}

The patient was a 7 year old Bedouin girl admitted to our hospital with a three week history of fever and limp. She is the eldest of four children in a typical healthy Bedouin family. They denied consumption of any dairy product from goats or sheep or contact with these animals. On physical examination she presented as a thin girl on the third percentile for weight. Her temperature was $40^{\circ} \mathrm{C}$, pulse $110 / \mathrm{min}$, blood pressure $90 / 60 \mathrm{mmHg}$. The spleen and liver were palpable 3 and $4 \mathrm{~cm}$ and the liver $4 \mathrm{~cm}$ below the costal margin respectively. Weight bearing and passive movements of the right hip were extremely painful. The right hip was held in flexion and its range of motion was limited. The rest of the physical examination was unremarkable. Laboratory studies showed the following: haemoglobin 116 $\mathrm{g} / \mathrm{l}$; white blood cell count $7.9 \times 10^{9} / 1$ with $13 \%$ band forms, $35 \%$ segmented, $45 \%$ lymphocytes, $5 \%$ monocytes, $1 \%$ basophils and $1 \%$ eosinophils. The platelet count was $198 \times 10^{9} / 1$; erythrocyte sedimentation rate $45 \mathrm{~mm} / \mathrm{h} ; \mathrm{C}$ reactive protein $100 \mathrm{mg} / \mathrm{l}$ antistreptolysin $\mathrm{O} 600$
IU; C3 $1.67 \mathrm{~g} / \mathrm{l} ;$ C4 $400 \mathrm{mg} / \mathrm{l}$. Tests for rheumatoid factor, antinuclear antibodies, and the standard agglutination test for brucella were negative. Figure 1 shows that plain radiographs of the hip were normal.

A technetium $99 \mathrm{~m}$ methylene diphosphate joint and bone scan showed increased radionuclide activity, in the early phase, over the hip joint and greater trochanter on the right, with a small photopenic area in the head of the femur. Aspiration of the right hip joint was performed and a small amount of fluid was found and sent for culture. Bone marrow aspiration showed no evidence of lymphoproliferative processes. As septic arthritis with possible osteomyelitis of the head of the femur was suspected, an open drainage of the right hip was performed and a small amount of turbid fluid with normal articular surfaces was found. In spite of parenteral treatment with cloxacillin, the patient remained pyrexial and toxic. Two weeks after admission, the bone marrow and synovial fluid cultures grew Brucella melitensis. The patient was subsequently treated with doxycillin by mouth for three weeks and intramuscular streptomycin for two weeks. The fever rapidly resolved and her general condition improved; the erythrocyte sedimentation rate decreased to $15 \mathrm{~mm} / \mathrm{h}$. However, follow up radiographs of the right hip were consistent with avascular necrosis of the right femoral head (figs 2 and 3 ).

In spite of bed rest and a non-weight bearing regimen the femoral head gradually collapsed. After six weeks in hospital she was discharged but still needed crutches. On follow up visits at $3,6,12$, and 18 months the patient remained well but a mild limp and limitation of motion in the right hip persisted.

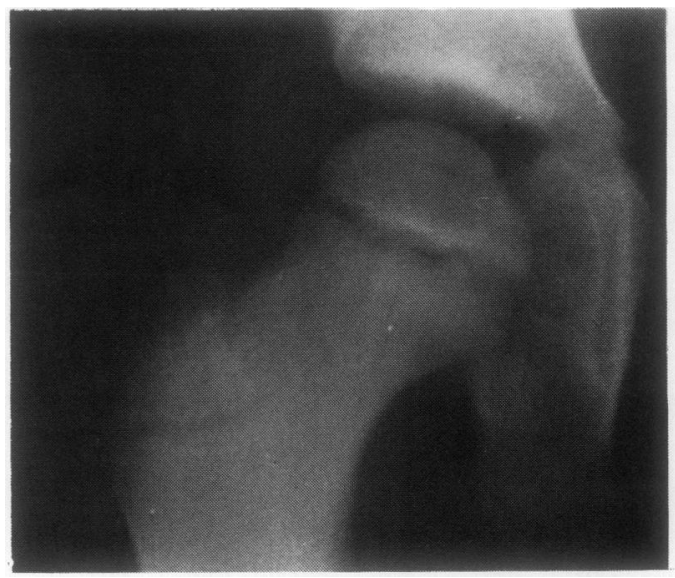

Figure 1 Anteroposterior view of the affected hip on the day of admission. 

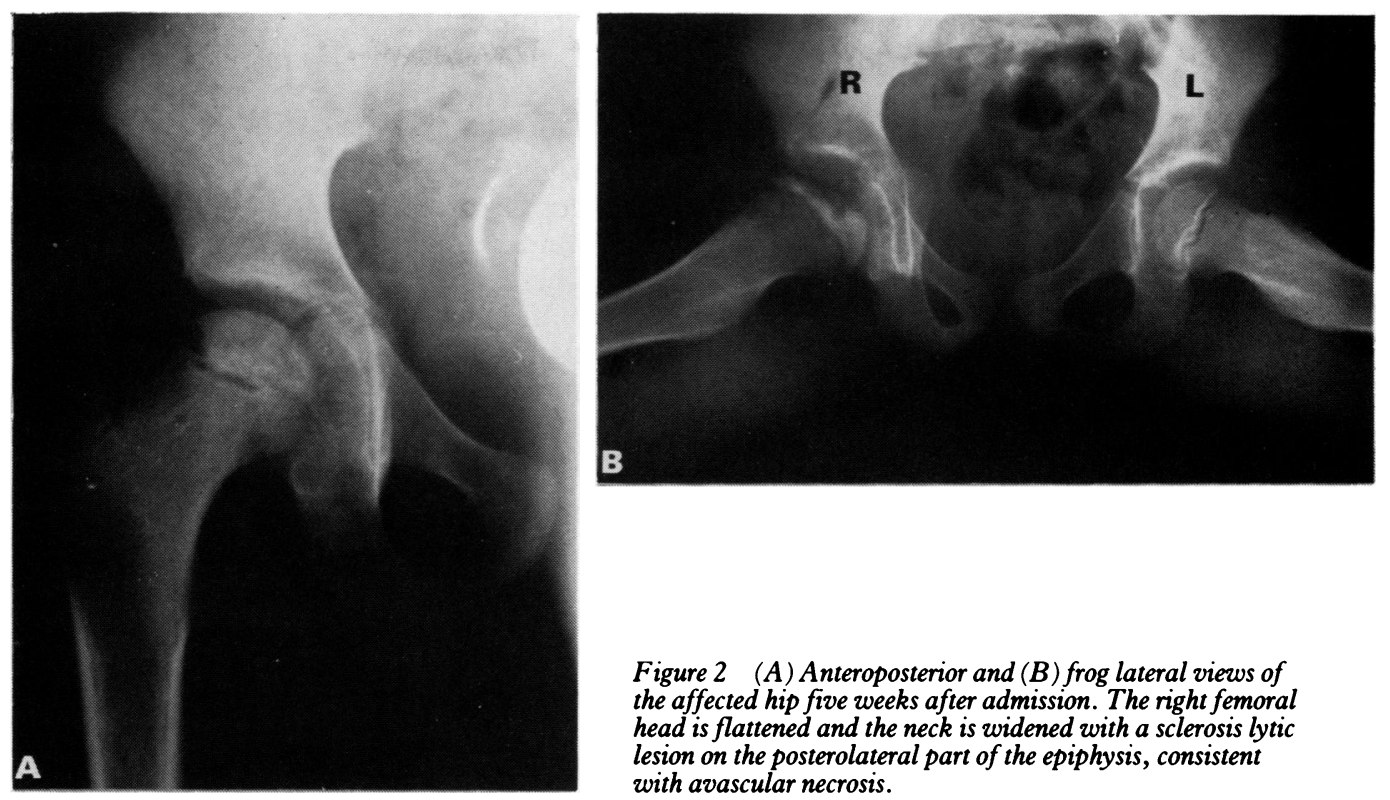

Figure 2 (A) Anteroposterior and (B) frog lateral views of the affected hip five weeks after admission. The right femoral head is flattened and the neck is widened with a sclerosis lytic lesion on the posterolateral part of the epiphysis, consistent with avascular necrosis.
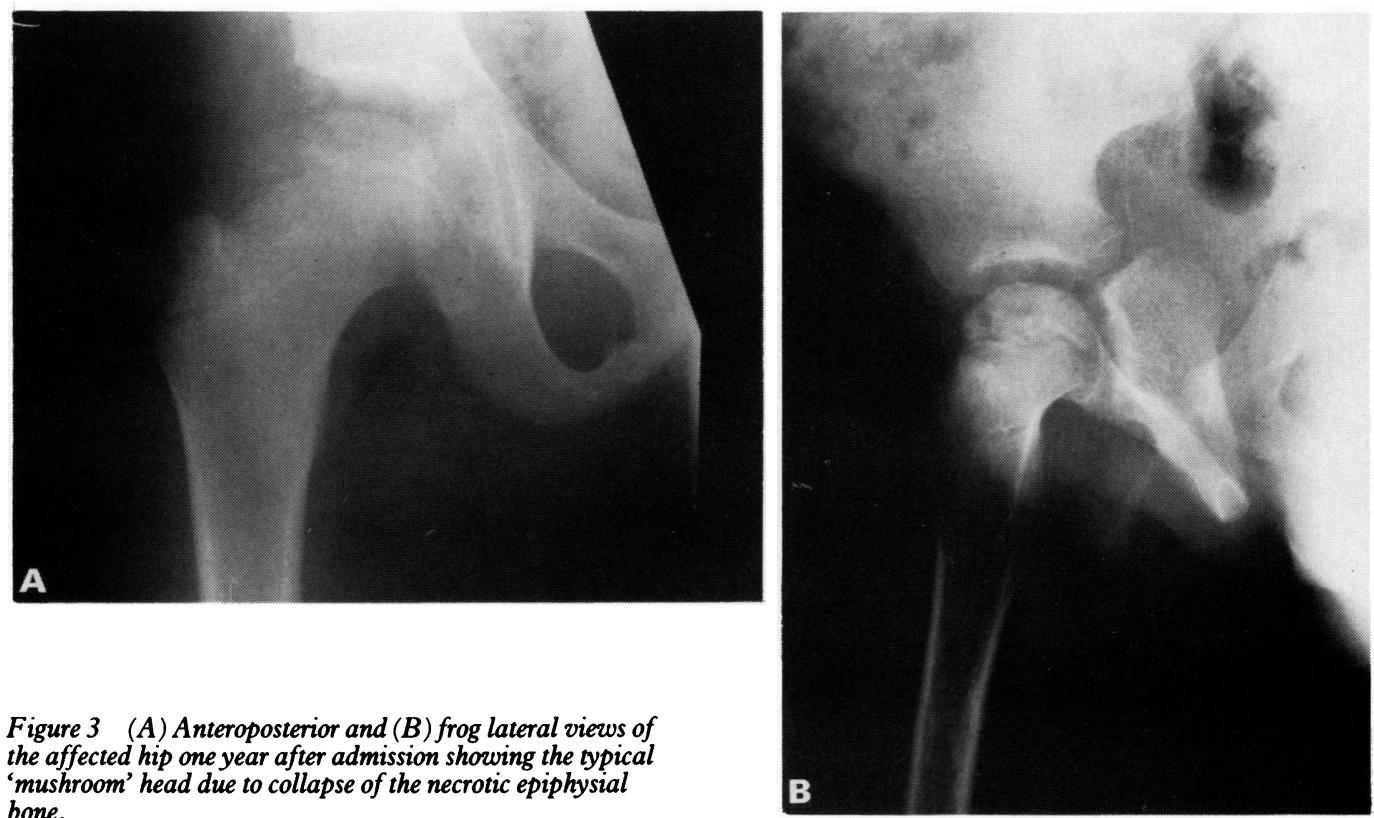

Figure 3 (A) Anteroposterior and $(B)$ frog lateral views of the affected hip one year after admission showing the typical 'mushroom' head due to collapse of the necrotic epiphysial bone.

\section{Discussion}

Osteoarticular manifestations, including arthritis, spondylitis, sacroiliitis, osteomyelitis, tendinitis, and bursitis occur in $30-85 \%$ of patients with brucellosis. Arthritis is the most common and affects lower leg joints, occurring mainly in children and young adults with acute disease. ${ }^{4}$ Similarly, in the Negev region of Israel, peripheral arthritis is the most common osteoarticular manifestation in Bedouin children with brucellosis. ${ }^{5}$ Sacroiliitis usually occurs in young adults simultaneously with the peripheral arthritis.

Spondylitis affecting the lumbar spine commonly occurs in the elderly and those patients with chronic brucellosis. ${ }^{6}$ In contrast to peripheral arthritis and sacroiliitis, spondylitis is generally related to bacterial seeding and may be associated with destruction of the inter- vertebral structures. Pyogenic infection of the hip joint, especially when treatment has been delayed for more than a few days, has a poor prognosis. On the other hand, sequelae following peripheral arthritis caused by brucella has rarely been reported. ${ }^{8}$ In two large series of patients with brucellosis with osteoarticular participation, no articular destruction was noted. ${ }^{79}$

Avascular necrosis of the head of the femur has been noted to occur either as an idiopathic process (Legg-Perthes disease), or as a complication of septic arthritis, toxic synovitis and osteomyelitis. In patients with brucella arthritis the association of photopenic lesions with poor outcome is unknown. A decreased tracer activity on a bone scan was only detected in the knee of a 5 year old child among 140 brucellosis patients with osteoarticular participation. No articular damage could be shown on a sub- 
sequent radiograph, however. ${ }^{10}$ Unfortunately, in the patient described in this report, the affected hip suffered permanent damage. The patient was noted to have a photopenic area in the femoral head prior to surgery and the radiological changes which occurred over the following months were very similar to those seen in Legg-Perthes disease. Whether this was avascular necrosis due to the intra-articular pressure produced by the infection or by direct participation of the bone remain unclear.

1 Gross E M, Torok V, Arbeli Y, et al. Human brucellosis in the Negev region of Israel. Isr f Med Sci 1982; 18: 972.

2 Gedalia A, Watemberg N, Rotschild M. Childhood brucellosis in the Negev region of Israel: clinical and epidemiological aspects. Harefuah 1990; 119: 313-5.

3 Del Rio M A. Brucella osteomyelitis. Pediatr Infect Dis f 1983; 2: 50-2.

4 Alarcon G S, Bocanegra T S, Gotuzzo E, et al. The arthritis of brucellosis: a perspective one hundred years after Bruce's discovery. I Rheumatol 1987; 14: 1083-5.

5 Gedalia A, Watemberg N, Rotschild M. Joint involvement of Gedalia A, Watemberg N, Rotschild M. Joint involvement
childhood brucellosis. Arthritis Rheum 1988; 31: S119.

6 Gutuzzo E, Alarcon G S, Bocanegra T S, et al. Articular involvement in human brucellosis: a retrospective analysis of 304 cases. Semin Arthritis Rheum 1982; 12: 245-55.

7 Lifeso R M, Harder E, McCorkell S J. Spinal brucellosis. f Bone foint Surg [Br] 1985; 67: 345-51.

8 Seal P V Morris C A. Brucellosis of the carpus. A case report. $\mathcal{F}$ Bone foint Surg [Br] 1974; 56: 327-30.

9 Abdul Rachman M, Mousa S A, Muhtaseb D S, et al. Osteoarticular complication of brucellosis: a study of 169 cases. Rev Infect Dis 1987; 9: 531-43.

10 Madkour M M, Sharif H S, Abed M Y, et al. Osteoarticular Actigraphy in 140 patients. Am f Roentgenol 1988; 150: 1101-5. 